\title{
DETECTING WALKABLE PLANE AREAS BY USING RGB-D CAMERA AND ACCELEROMETER FOR VISUALLY IMPAIRED PEOPLE
}

\author{
Kenta Imai ${ }^{1}$, Itaru Kitahara ${ }^{2}$, Yoshinari Kameda ${ }^{2}$ \\ ${ }^{1}$ Graduate School of Systems and Information Engineering, University of Tsukuba \\ ${ }^{2}$ Center for Computational Sciences, University of Tsukuba
}

\begin{abstract}
When visually impaired person has to walk out, they have to use white canes, but the range that can be scanned by a white cane is not long enough to walk safely. We propose to detect walkable plane areas on road surface by using the RGB-D camera and the accelerometer in the tablet terminal that is attached to the RGB$\mathrm{D}$ camera. Our approach can detect plane areas in longer distance than a white cane. It is achieved by using height information from the ground and normal vectors of the surface calculated from a depth image obtained by the RGB-D camera in real time.
\end{abstract}

Index Terms - visually impaired people, accelerometer, plane detection, depth image, white cane

\section{INTRODUCTION}

In 2014, the World Health Organization reported that the number of visually impaired people was estimated to be approximately 285 million worldwide [1]. When a visually impaired person has to walk out, he/she usually detects walkable plane areas by using a white cane. The range that can be scanned by a white cane is about one or two steps. White canes are not suitable to detect obstacles and/or steps far from the user. In addition, small holes and lower steps are hard to detect by scanning of the white cane. Visually impaired people need to detect walkable plane areas in a longer and wider range so that they feel safer on walking out.

Researches and developments of walking support systems that have higher functions than a white cane have been proposed [2][3][4]. They analyze depth information obtained by a laser scanner or a Microsoft Kinect. In our research, we utilize Intel R-200 RGB-D camera because it can always capture depth images both in indoor and outdoor scenes.

We propose a new detection method of walkable plane areas on road surface by using the R-200 and an associated accelerometer. Our implemented preliminary system can detect plane areas in longer distance than a white cane. The advantage of our proposed method is to detect irregular areas of which height is upper or lower of the walkable plane level, which cannot be done by simple scanning by a white cane.

In our approach, the R-200 is set at the height of a user chest so that it can cover wide range of frontal area of the user. The accelerometer is rigidly attached to the $\mathrm{R}-200$. The walkable plane area is detected by extracting horizontal plane areas based on the real time analysis of the camera orientation.

We have built a prototype system in which the R-200 is connected to a Microsoft tablet terminal Surface 4 that includes an accelerometer. We confirmed that our approach is available both in indoor and outdoor scenes with obstacles and upper/lower steps.

\section{RELATED WORKS}

As for intelligent systems that utilize sensors for visually impaired people, Vere et al. [2] propose the way of detecting obstacles by using a smartphone and a laser pointer. In their system, the laser pointer beam reflection is recognized by the smartphone camera. The distance from the laser pointer to reflection surface is computed by active triangulation. However, using this system may make visually impaired people walking more slowly than using a white cane. Takizawa et al. [3] propose the system that integrates a white cane and a Microsoft Kinect. They install the Kinect on the white cane and edges in a depth image obtained by the Kinect is detected. Based on the edge information, they succeed in detecting a floor, obstacles and/or stairs. However, the Kinect may not work well in outside scenes. Sonda et al. [4] propose the way of detecting obstacles or stairs by using an ultrasonic sensor and a monocular camera. The distance can be estimated only one line at a time, but it can identify objects by using SVM with a RGB image analysis. This approach is not good for detecting a horizontal plane surface because they need to sweep the frontal areas by their system.

\section{DECTION OF A WALKABLE PLANE AREA ON ROAD SURFACE}

We propose the method to detect walkable plane areas by using the R-200 and the accelerometer in real time. The R-200 is set at the height of a user chest and it is directed to downward slightly so that its field of view covers the road surface in the frontal area of the user. Since the R-200 does not have an accelerometer in its inside, we connect the R-200 with the Surface 4 and read the accelerometer in the Surface 4.

Our basic strategy is to detect plane areas of which the surface normal is parallel to the gravity direction by estimating the orientation of the R-200 from the status of the accelerometer. Then, only the ground level planes are extracted by estimating their height. The overall procedure is shown in Figure 1.

First, the depth image of the R-200 is converted into point cloud form. By referring the accelerometer, one axis of the coordinate system of the point cloud can be parallel to the gravity direction. Since the height of the R-200 to the ground is static due to the mounting point of the R-200, the height of each point in the point cloud can be calculated.

At the same time, the normal vector of each point in the point cloud is estimated. In order to pursue calculation efficiency, the points in the cloud are segmented by small rectangular areas at the view of R-200. The rectangular areas of which the height is close to the ground level and the surface normal is parallel to the gravity direction are counted as walkable plane areas. 


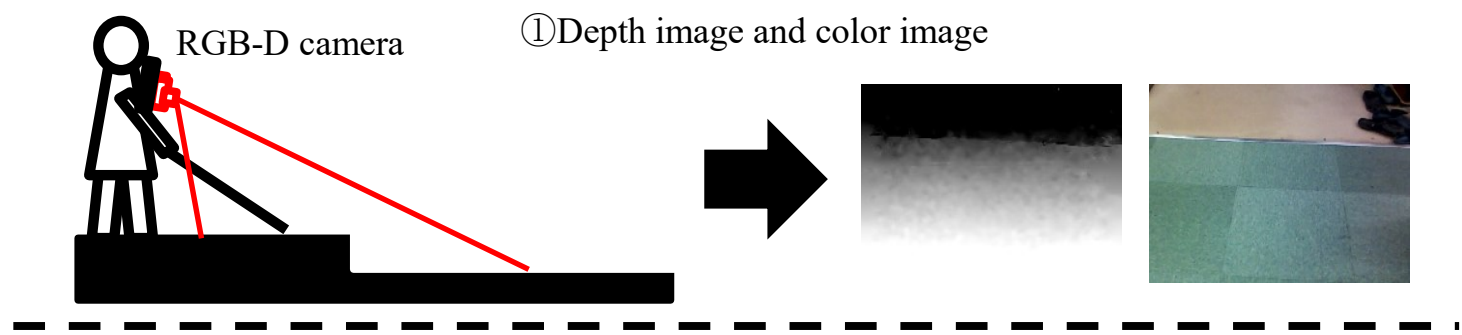

(2)Point cloud

(3) Plane detection
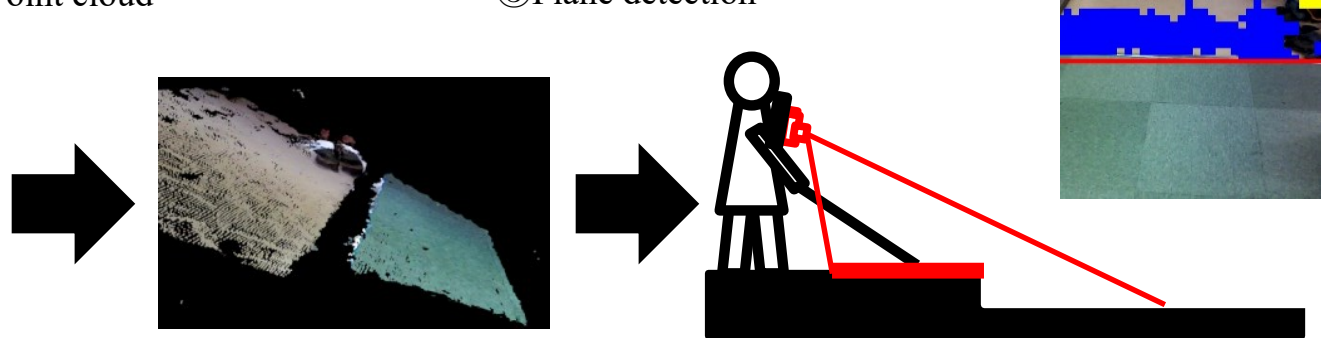

Figure 1. Procedure overview

Finally, none-walkable plane areas are classified into upper or lower areas and the distance to the closest none-walkable plane areas is estimated. It is the limit of the safe region to walk.

\section{CALCULATION OF HEIGHT AND NORMAL VECTOR}

\subsection{Height}

In order to estimate the height of the points along the gravity direction, we introduce a Cartesian coordinate system of which one axis is parallel to the gravity direction and another axis is set to the horizontal walking direction of the user, i.e., direction of the body orientation. We call the axes the gravity axis and front axis respectively.

The original depth image from the R-200 has depth information from the camera center at each pixel. Figure 2 shows a color image of a floor on the left and its depth image on the right. Note that the brighter intensity in the depth image corresponds to shorter distance from the camera, and the viewing angle of the two images corresponds to the same area in the frontal area of the R-200. A profile of the depth image taken at the red line in Figure 2 is shown in the upper graph of Figure 3. The horizontal axis in the graph corresponds to the pixel position along the red line from the top to the bottom. Since the R-200 is slightly directed downward at angle $\theta$, and the pixel position is not linearly mapped to the position along the front axis, the profile at the red line forms a curve in the graph.

Since what we need is to estimate the ground level of the observed surfaces in the depth image, the height of the pixels are calculated based on the angle $\theta$. The lower graph of Figure 3 shows the calculation result of the red line along the gravity axis.

The graph shows the target surface (floor) is at constant height along the profile, and it implies the surface forms a plane at the height of about $1050 \mathrm{~mm}$.

Currently, our prototype system assumes that the R-200 is set along the front axis. It means no twisting rotation is allowed around optical axis of the R-200. This limitation will be easily removed by introducing the second angle parameter $\phi$ from the accelerometer.

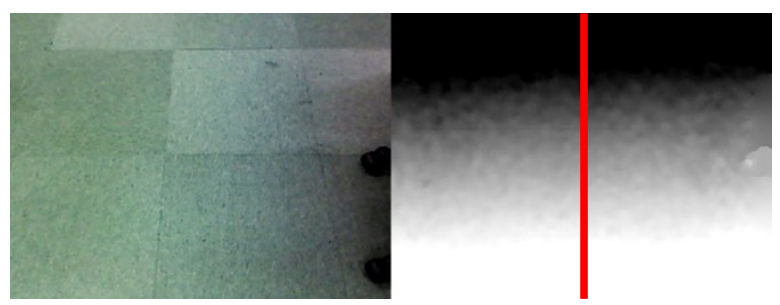

Figure 2. Color image and depth image

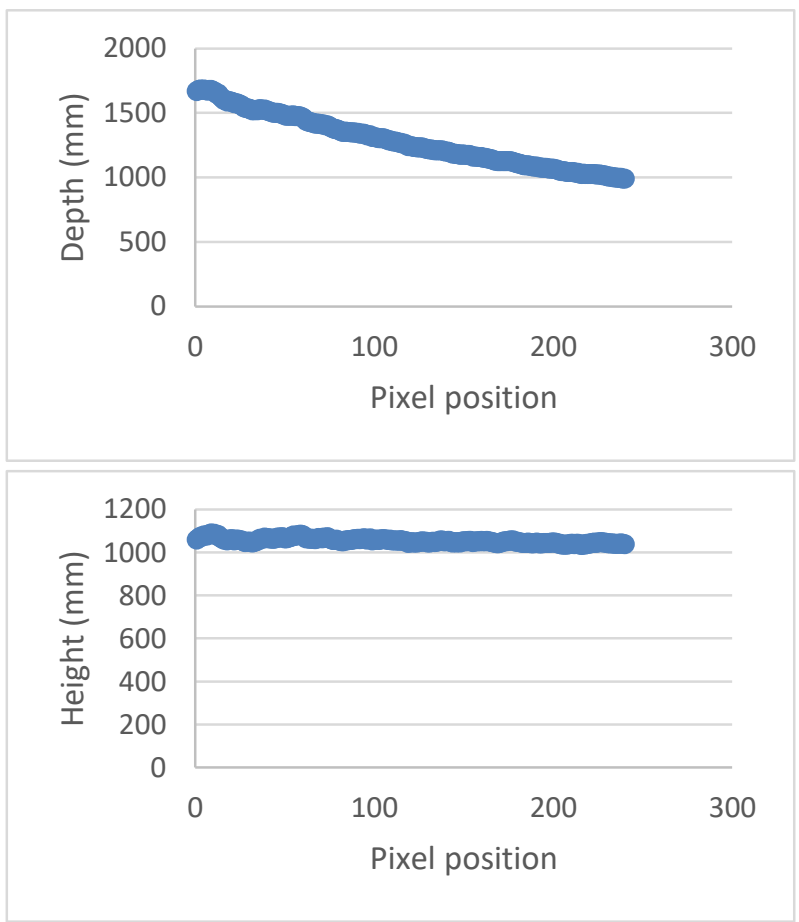

Figure 3. Calculating height in depth image

\subsection{Normal vector}

We use the approach of S.Holzor[5] to estimate normal vectors at high speed for the obtained depth image. The estimation may 
not be good at complicated surfaces in a scene, but it will not be a serious problem as we use normal vectors to check a small area is flat or not.

\subsection{Walkable plane by small rectangular areas}

We detect walkable plane areas by setting a threshold on the calculated height and the normal vectors. In our approach, the points in the point cloud are segmented into same size of small rectangular areas as shown in Figure 4 right. The segmentation is conducted to reduce the influence of the noise in depth images.

We first calculate the height average and the averaged vector of the surface normals in each small rectangular area. If the height is close to the assumed height of the road surface, and if the averaged surface normal is closely parallel to the gravity axis, the rectangular area is considered to be a part of walkable plane area. Other small rectangular areas are treated as non-road surface areas.

A snapshot of the process is shown in Figure 4. The left image is the RGB image taken by the R-200 and the right shows the result of the process over the color image. Red rectangles indicate the size of the segmentation process. The rectangles with original texture are detected as walkable plane area, whereas the yellow rectangles are recognized as upper non-road surface areas, of which the height is higher than the ground level, and the blue rectangles lower non-road surface areas.

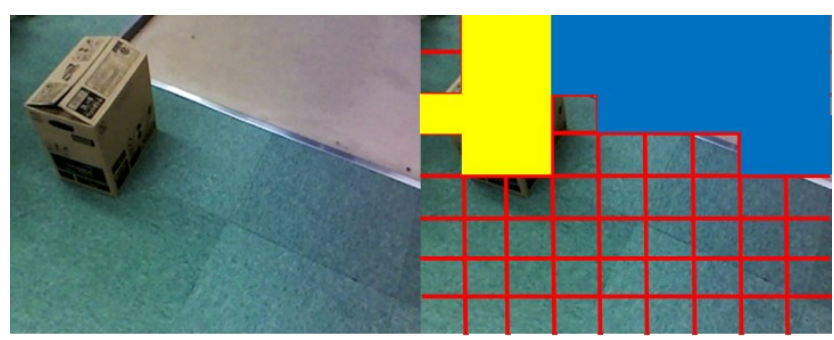

Figure 4. Detection of walkable plane areas

\subsection{Noise removal by referring inter frames}

Noise may be found in depth images from the R-200. As a tendency of noise included in the depth images, temporal continuity seldom happens. Fault detection may happen in a frame, but it will not occur at the same rectangle areas in the next frame Because we assume the system will be used on walking, obstacles and steps should be found continuously over frames at close rectangle areas. Since our prototype system can process depth images at around $20 \mathrm{fps}$, we remove noise affection by introducing this discontinuity assumption.

If a small rectangular area is determined as a candidate of nonroad surface area, the four-connected next rectangles in the previous frame are checked and if at least one of them are also marked as non-road surface area, it will be marked as so. If not, it is treated as a part of walkable plane area.

\subsection{Horizontal distance to the closest non-road surface}

The shortest horizontal distance to the end of the walkable planner area is estimated by finding the closest non-road surface rectangle areas along the front axis. It can be calculated based on the orientation of the R-200 against the world.

\section{EXPERIMENT}

\subsection{Experiment setting}

In order to evaluate our approach, we detected walkable plane areas on multiple roads both in indoor and outdoor scenes by using our prototype system. The R-200 has two different depth imaging system for indoor scene and outdoor scene. In indoor scene, it emits infrared light and uses one camera to produce depth image actively. In outdoor, it uses two cameras to produce depth image passively. The measurable range of depth image of the $\mathrm{R}-200$ is $0.5 \mathrm{~m}$ to $4.0 \mathrm{~m}$.

In this experiment, we held a Surface 4 and fixed the R-200 at a height of about $110 \mathrm{~cm}$. For each experiment place, we estimated the distance to the non-road surface candidate area that is the closest to the user, and evaluate the performance by comparing it with the true distance. The places include a descending step, an ascending step, and obstacles both in indoor and outdoor scenes. The CPU of Surface 4 is Intel Core i7 $2.20 \mathrm{GHz}$, and the frame rate during this evaluation was about 20 fps.

\subsection{Experiment results}

We show experiment results in Figure 5. Three places are shown from the top to the bottom in Figure 5. They correspond to the descending step, the ascending step, and the obstacle. The left part corresponds to indoor scenes, and the right the outdoor scenes.

At each place, the left side is the obtained color image, the right side is the result image which is shown in the same manner of Figure 4. A red line in the result image represents the border of the non-road surface area.

The descending step (indoor, Figure 5 upper left):

There is a step of about $6 \mathrm{~cm}$ low ahead. Our approach detected the road surface after the step as a non-road surface area. The estimated horizontal distance to the red line was $134 \mathrm{~cm}$. The actual distance from the user to the step is $140 \mathrm{~cm}$.

The ascending step (indoor, Figure 5 left middle):

There are ascending stairs of about $10 \mathrm{~cm}$ height at each step. Our approach detected the staircase and the handrail portion of the staircase as a non-road surface area, and the estimated horizontal distance to the red line was $130 \mathrm{~cm}$. The actual distance from the user to the step is $135 \mathrm{~cm}$.

The obstacle (indoor, Figure 5 down left):

There is an obstacle of about $20 \mathrm{~cm}$ height in front. Our approach detected the obstacle as a non-road surface area, and the estimated horizontal distance to the red line was $160 \mathrm{~cm}$. The actual distance from the user to the step is $165 \mathrm{~cm}$.

The descending step (outdoor, Figure 5 upper right):

There is a descending staircase of about $12 \mathrm{~cm}$ height in each step in front. In the figure, we cannot confirm the second and subsequent steps. Our approach detected the border where the lower staircase starts as the edge of a non-road surface area. The estimated horizontal distance to the red line was $161 \mathrm{~cm}$. The actual distance from the user to the step is $165 \mathrm{~cm}$.

The ascending step (outdoor, Figure 5 right middle):

An ascending staircase of about $10 \mathrm{~cm}$ in each step starts from back of the latticed drain channel lid. Our approach detected the place beyond the stairs as a non-road surface 
area. The estimated horizontal distance to the red line was $200 \mathrm{~cm}$. The actual distance from the user to the step is $210 \mathrm{~cm}$.

The obstacle (outdoor, Figure 5 down right):

There are arch shaped car-stops ahead. Our approach detected them as non-road surface areas. The estimated horizontal distance to the red line was $165 \mathrm{~cm}$. The actual distance from the user to the stop is $170 \mathrm{~cm}$.

We have conducted verification experiments in other various situations. Expected results were obtained within the range from about $1 \mathrm{~m}$ to $2 \mathrm{~m}$. This result means that our approach can detect up to longer distance than a white cane to detect steps and obstacles. When the range becomes $2 \mathrm{~m}$ to $3 \mathrm{~m}$ or more, there is a tendency for the depth image to contain more noise and the system showed unstable results. In order to clear this problem, we consider the way of removing noise in depth images by using the texture consistency in a color image.

\section{CONCLUSION}

Based on our proposed approach, we successfully detected walkable plane areas by using the R-200 and the accelerometer to cover a longer range than a white cane. Our preliminary system can detect not only obstacles and steps upward but also steps downward, which is not easy to detect by a white cane.

We confirmed that our approach achieved our initial goal through the experiments both in indoor and outdoor scenes. We need to demonstrate the validity of our system for visually impaired people. We will verify precision of the detection of steps and obstacles more precisely. Our next research work is to extend the detection distance longer and design a new UI to effectively notify the limit of walkable plane area to the users. We will use sounds to notify the user of the limit of walkable plane
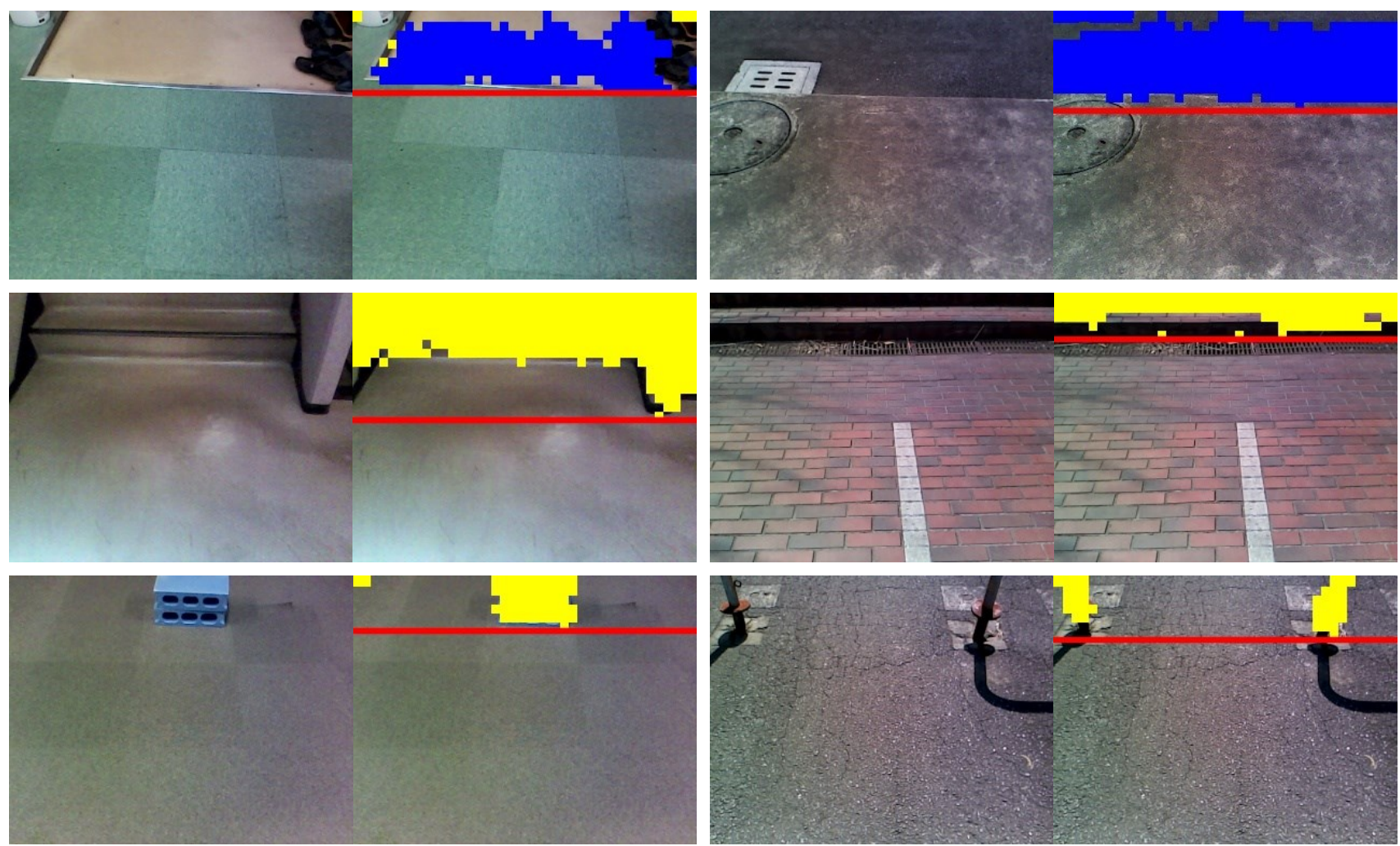

area. Since visually impaired people usually understand the situation of their surrounding areas by surrounding sounds when walking, we will consider the way of notifying as simple as possible not to interfere surrounding sounds. We thank the support of JST-RISTEX and KAKENHI $17 \mathrm{H} 01773$.

\section{REFERENCES}

[1] World health organization, media centre, visual impairment

[2] P. Vera, D. Zenteno, and J. Salas, "A smartphone-based virtual white cane," Pattern Analysis and Applications, vol. 17, pp. 623-632, Aug. 2014.

[3] H. Takizawa, S. Yamaguchi, M. Aoyagi, N. Esaki, and S. Mizuno, "Kinect cane:an assistive system for the visually impaired based on the concept of object recognition aid," Personal and Ubiquitous Computing, vol. 19, No. 5, pp. 995-965, 2011.

[4] S. Bouhamed, I. Kallel, and D. Masmoudi, "New electronic white cane for stair case detection and recognition using ultrasonic sensor," International Journal of Advanced Computer Science and Applications, vol. 4, No. 6, 2013.

[5] S. Holzer, R. B. Rusu, M. Dixon, S. Gedikli, and N. Navab, "Adaptive neighborhood selection for real-time surface normal estimation from organized point cloud data using integral images," Intelligent Robots and Systems, pp. 2684 $2689,2012$. and blindness, fact sheet no. 282. Accessed 1 Aug 2014

Figure 5. Result of detections by using our approach (upper left: the indoor descending step, left middle: the indoor ascending step, down left: indoor obstacle, upper right: the outdoor descending step, right middle: the outdoor ascending step, down right: outdoor obstacle) 